\title{
La tradizione testuale del Lachete di Platone. Rapporti stemmatici fra i manoscritti della terza famiglia
}

Maria Vittoria Curtolo

(Università di Pisa; Universität Hamburg)

\section{The textual tradition of Plato's Laches. Stemmatic relationships between the manuscripts of the third family}

\begin{abstract}
Thirty-three manuscripts preserve the complete text of Plato's Laches. Three of them, Vindobonensis Suppl. gr. 7 (siglum W), Lobcovicianus (Roudnice VI Fa 1; siglum L) and Vaticanus graecus 1029 (R), belong to the same family, i.e. the third family. Despite previous discussion, today the date of the most ancient section of $\mathrm{W}$ is assigned to saec. XI. The independence of this manuscript in the first seven tetralogies was stated for the first time by Josef Král in $1892^{1}$ and the other manuscripts of its family derive - directly or indirectly - from it. This paper will give an overview of the extant manuscripts of the third family and investigate the mutual relationships between them, as they result from the collation of all thirty-three manuscripts of the Laches.
\end{abstract}

\section{Keywords}

Plato; Laches; fifth tetralogy; textual criticism; manuscripts; textual tradition; third family; Vind. Suppl. gr. 7; W; Lobcovicianus; Vat. gr. 1029; Esc. y. I. 13; Anonymus K

1 Král (1892). 


\section{Introduzione}

Il Lachete di Platone è tràdito integralmente da trentatré manoscritti, ai quali si aggiungono alcuni codici di excerpta. ${ }^{2}$ Altre fonti del testo del dialogo sono i papiri, le testimonianze indirette, le traduzioni latine e le prime edizioni a stampa.

Come per gli altri dialoghi della quinta tetralogia - Carmide, Teage e Liside - l'oxoniense Bodl. E. D. Clarke 39 (siglum B; a. 895), il veneziano Marc. App. Class. IV, 1 (coll. 542) (siglum T; sec. X) e il Vind. Suppl. gr. 7 (W; sec. XI) sono fonti primarie del testo del dialogo. ${ }^{3}$ Secondo D. J. Murphy anche il Par. gr. 1813 (sec. XIII; siglum Q) sarebbe un testimone indipendente del testo del Lachete, capostipite di una quarta famiglia. ${ }^{4}$ In questa sede tuttavia non ci si soffermerà sulla collocazione del Parigino nel quadro della tradizione platonica. ${ }^{5}$

L'attenzione di questo contributo è rivolta alla terza famiglia dei manoscritti che tramandano il Lachete - W, il Lobcoviciano (Roudnice VI Fa 1; siglum L) e il Vat. gr. 1029 (R) - e ai rapporti che intercorrono tra di essi. ${ }^{6}$

\section{Il capostipite W}

Il nucleo di W (Wien, Österreichische Nationalbibliothek, Suppl. gr. 7; La. 482r-494r) contiene il Prologo di Albino, le tetralogie 1-3 e, in un ordine inusuale, i dialoghi dall'Alcibiade I al Menesseno, escludendo però l'Alcibiade II. ${ }^{7}$ A lungo si è discusso sulla datazione di questo codice, con proposte che si estendevano dal 950 al sec. XII. ${ }^{8}$ La collocazione nel sec. XI è stata suggerita da L. A. Post e da A. Carlini; G. Cavallo propende per la fine

2 Per la ricognizione dei codici contenenti il Lachete punto di avvio sono stati Post (1934: pp. 65-92); Wilson (1962); Brumbaugh \& Wells (1968: p. 94); Brumbaugh (1990); il database Pinakes.

3 Per alcune indicazioni bibliografiche sui tre codici si rinvia a Brockmann (1992: p. 25 per B; p. 34 per T; pp. 35-36 per W). Le tradizioni testuali di Carmide, Teage e Liside sono state studiate rispettivamente da Murphy (1990), Joyal (1998) e Martinelli Tempesta (1997).

4 Murphy (1994).

$5 \quad$ Sullo status di Q nella tradizione di altri dialoghi cfr. Duke (1991) per il Fedone; Murphy \& Nicoll (1993) per il Cratilo; Moreschini (1965: pp. 173-174) per il Fedro; Murphy (1992a) per Fedro, Alcibiade II e Ipparco; Carlini (1964: p. 27) per Alcibiade II e Ipparco; Murphy (1990: pp. 334-338), (2002: pp. 132-148) per il Carmide. Cfr. anche Post (1934: p. 83); Duke \& Hicken \& Nicoll \& Robinson \& Strachan (1995: Praef., pp. IX-XI); Pontani (1997: pp. 109-110, spec. n. 38); Berti (2001: p. 365, n. 50).

6 In questo contributo non verranno esaminati i ridotti estratti del Lachete tràditi dal Pal. gr. 173 (cfr. Menchelli 1991: p. 106). Scheda catalografica del Palatino in Stevenson (1885: p. 91).

7 Informazioni codicologiche e paleografiche su W in Hunger (1957: pp. 13-14); Hunger \& Hannick (1994: pp. 12-16) e Mazal (1981: p. 313), ai quali si rinvia anche per approfondimenti bibliografici. Per la storia del codice cfr. Carlini (1992: pp. 13-21). Cfr. anche Boter (1989: pp. 61-62); Jonkers (2017: pp. 88-89). Di recente le relazioni stemmatiche fra i testimoni della terza famiglia dell'Eutifrone sono state studiate da Manfrin (2017).

8 P. Maas (apud Dodds 1959: p. 39) propose la datazione alta (c. 950-1050). Sintesi delle varie posizioni in Nicoll (1966: p. 70); Perria (1983-1984: pp. 99-100); Martinelli Tempesta (1997: p. 127); Joyal (1998: p. 35). Cfr. anche Murphy (1995a: pp. 159-161). 
del secolo e L. Perria, che ha riconosciuto nella mano di W il copista del Vat. gr. 407, per gli anni 1050-1075. ${ }^{9}$ Lo scriba del nucleo di W è stato chiamato da G. Prato 'Anonimo א', «dalla lettera che per la sua caratteristica forma consente di riconoscerne la scrittura a prima vista». ${ }^{10}$

Oltre a questa parte più antica, si individuano nel codice altre due sezioni vergate da due differenti mani, collocabili entrambe nel sec. XIII: W2, a cui si devono Clitofonte, Repubblica e Timeo, e W3 che ripristinò i fogli caduti di Teeteto (f. 139), Simposio (f. 256) e Lachete (ff. 486-488 = La. 1865d11-191b3 fino ad av̉tòv) e aggiunse il De natura mundi et animae di Timeo Locro. ${ }^{11}$ Per quest'ultimo Marg provò che la fonte di W3 è l'Escorialense y. I. 13 post correctionem, ${ }^{12}$ ragion per cui A. Carlini ha suggerito che il medesimo codice sia il modello anche per i fogli reintegrati dei dialoghi platonici e D. J. Murphy, indipendentemente, ha confermato la dipendenza di W3 da Esc. y. I. 13 per i fogli di Simposio e Lachete.$^{13}$ Agli accordi tra Esc. e W3 ricordati da Murphy si può anche aggiungere:

$187 \mathrm{e} 7$

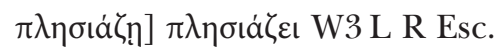

Il testo del Lachete che leggiamo oggi nel codice risale pertanto, quasi interamente (ff. 482r-485v, 489r-494v), alla seconda metà del sec. XI. Nel 1892 J. Král dimostrò che la sezione antica di W costituisce un testimone indipendente per la costituzione del testo. ${ }^{14}$ Pur non essendo l'indagine del rapporto di W con gli altri codici primari obiettivo di questo contributo, dalla collazione emerge una serie di convergenze in errore tra $\mathrm{T}$ e $\mathrm{W}$ contro B, quali ad esempio: ${ }^{15}$

9 Post (1934: p. 90); Carlini (1972: p. 171); Perria (1983-1984). G. Cavallo nella lettera ricordata da Carlini (1972: p. 171 n. 8): «le caratteristiche grafiche mi sembra suggeriscano quale data probabile la fine del secolo XI. Sarei comunque del parere di escludere senz'altro una data più alta; quanto all'attribuzione al secolo XII, essa resta probabile, ma mi lascia qualche perplessità».

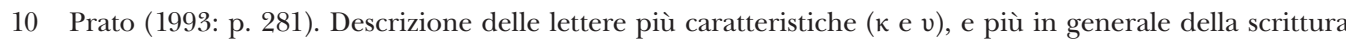
del copista (la base è una Perlschrift, con però evidenti innovazioni) in Perria (1983-1984: pp. 97-98, 100) e Perria (1992: pp. 124-126), cui si rinvia (pp. 120-124) anche per un'analisi degli aspetti codicologici della sua produzione. Oltre a Perria (1983-1984), sui manoscritti dell' 'Anonimo א' cfr. anche Perria (1990).

11 Cfr. la descrizione catalografica in Hunger \& Hannick (1994: pp. 12-15), in cui tuttavia si riconduce, per mero errore, W2 al 1200 circa. Hunger (1957: pp. 13-14) attribuiva i ff. 139, 486-488, a una mano del sec. XIV, non menzionando il foglio del Simposio successivamente reintegrato. Questo è stato individuato da Brockmann (1992: p. 237) e poi ricordato nel catalogo di Hunger \& Hannick (1994: pp. 14-15). Eleuteri (1993: p. 457) propone di collocare W2 e W3 tra il 1250 e il 1300.

12 Marg (1972: pp. 20-21). Scheda di catalogo dell'Esc. y. I. 13 in de Andrés (1965: pp. 190-191, nr. 306).

13 Carlini (1992: p. 30); Murphy (1992b: pp. 100-101, spec. n. 11). Sul modello di W3 per il foglio del Teeteto cfr. Murphy (1995a: pp. 156-158). Per recenti precisazioni sulla datazione di Esc. cfr. p.e. Murphy (1995a: pp. 158-159); Menchelli (2007: p. 175).

14 Negli Elenchi 1. e 2. non si riportano le lezioni già indicate da Král (1892: pp. 169, 173, 192). Le lezioni a 178a2-3, 180d5, 182b2, 192e3 sono evidenziate anche da Boter (1987: p. 154, nn. 32, 33).

15 Le lezioni sono ricavate dalla collazione integrale del Lachete che ho condotto sui manoscritti che lo tramandano. Si riportano negli apparati le varianti degli apografi, se divergenti dai codici primari. Benché, come detto sopra, non si intenda esaminare in questa sede lo status di Q nella tradizione del Lachete, si indicano, per completezza, negli apparati anche le lezioni di questo codice. 


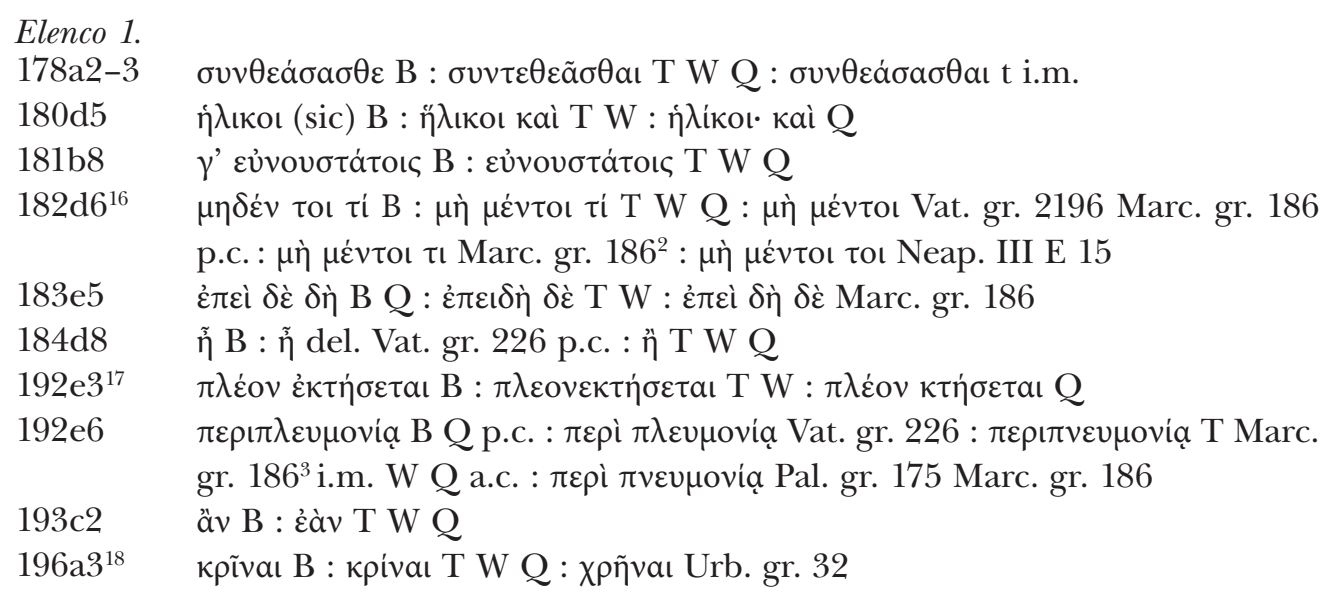

Alcune lezioni sono tràdite da T contro accordi di B e W:

Elenco 2.

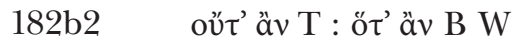

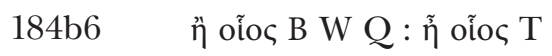

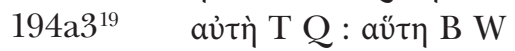

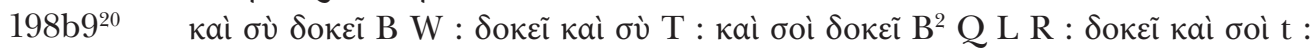
кaì $\sigma u v \delta о \kappa \varepsilon \tilde{~ s c r i p s i t ~ B u r n e t ~}$

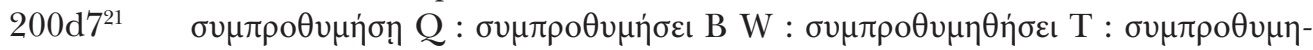
$\theta$ ө̇ọ Par. gr. 1808

A 182b2 e 194a3 il Marciano potrebbe aver ristabilito congetturalmente la lezione corretta. A 184b6 Burnet accoglie la lezione proposta da Schleiermacher (oĩoc). ${ }^{22}$ Negli ultimi due casi dell'Elenco 2. T presenta una lezione erronea, differente tuttavia da quella - comunque scorretta - tràdita da B e W (inseritasi in quest'ultimo codice per contaminazione con il ramo di B?).

I due Elenchi suggerirebbero da un lato una congiunzione tra $\mathrm{T}$ e W, dall'altro l'indipendenza di $\mathrm{W}$ da $\mathrm{T}$ e quindi la discendenza dei due codici da un comune iparchetipo, come propongono anche G. Boter nella sua indagine sul comportamento di W all'inter-

16 Con Marc. gr. $186^{2}$ mi riferisco gli interventi di Demetrio Sguropulo (cfr. Mioni 1976: p. 305).

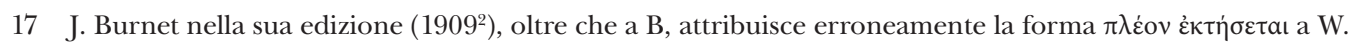

18 X riscontrano nel codice, almeno nel testo del Lachete. Per errori simili nel Liside cfr. Martinelli Tempesta (1997: pp. 18, 22-23).

19 Si dovrebbe correggere l'apparato di Burnet $\left(1909^{2}\right)$ che riporta aútì come lezione di W.

20 Con $\mathrm{B}^{2}$ si indicano gli interventi di Areta, la cui mano è stata riconosciuta nelle annotazioni del codice da E. Maass (1884). Cfr. Brockmann (1992: pp. 38-42). t si riferisce alle correzioni nel codice T attribuite a copisti diversi dal primo (cfr. p.e. Murphy 1990: p. 318; Martinelli Tempesta 1997: p. 29). In questo caso $\mathrm{T}$ non presenta la lezione corretta, ma un ordo verborum differente da quello di B W.

21 Cfr. Murphy (1994: p. 6).

22 Burnet $\left(1909^{2}\right)$. 
no della tradizione platonica e C. Brockmann nel suo studio sulla tradizione manoscritta del Simposio. Boter suggerisce che in alcuni dialoghi, tra cui il Lachete (p. 147), W e T risalgano a una stessa fonte, che sarebbe il perduto primo volume di A (Par. gr. 1807), in altri W si avvicini a B e in Teeteto e Sofista il Vindobonense sia autonomo dagli altri due codici. Brockmann indipendentemente è giunto alla stessa conclusione per il Simposio, tracciando uno stemma bipartito che vede B opporsi a un iparchetipo da cui dipendono da un lato T - e, per gli excerpta del dialogo che tramanda, il Pal. gr. 173 (P) - dall'altro W. ${ }^{23}$ Per il Lachete, inoltre, D. J. Murphy ha individuato alcuni passi che proverebbero che $\mathrm{B}$ derivi da una trascrizione minuscola diversa da quella che avrebbe originato $\mathrm{T}, \mathrm{W}$ e, se si accetta l'ipotesi della sua indipendenza, $Q \cdot{ }^{24}$

Alcune lezioni inferiori sono proprie di W e di due codici, il Lobcoviciano (L) e il Vat. gr. 1029 (R). A titolo esemplificativo: ${ }^{25}$

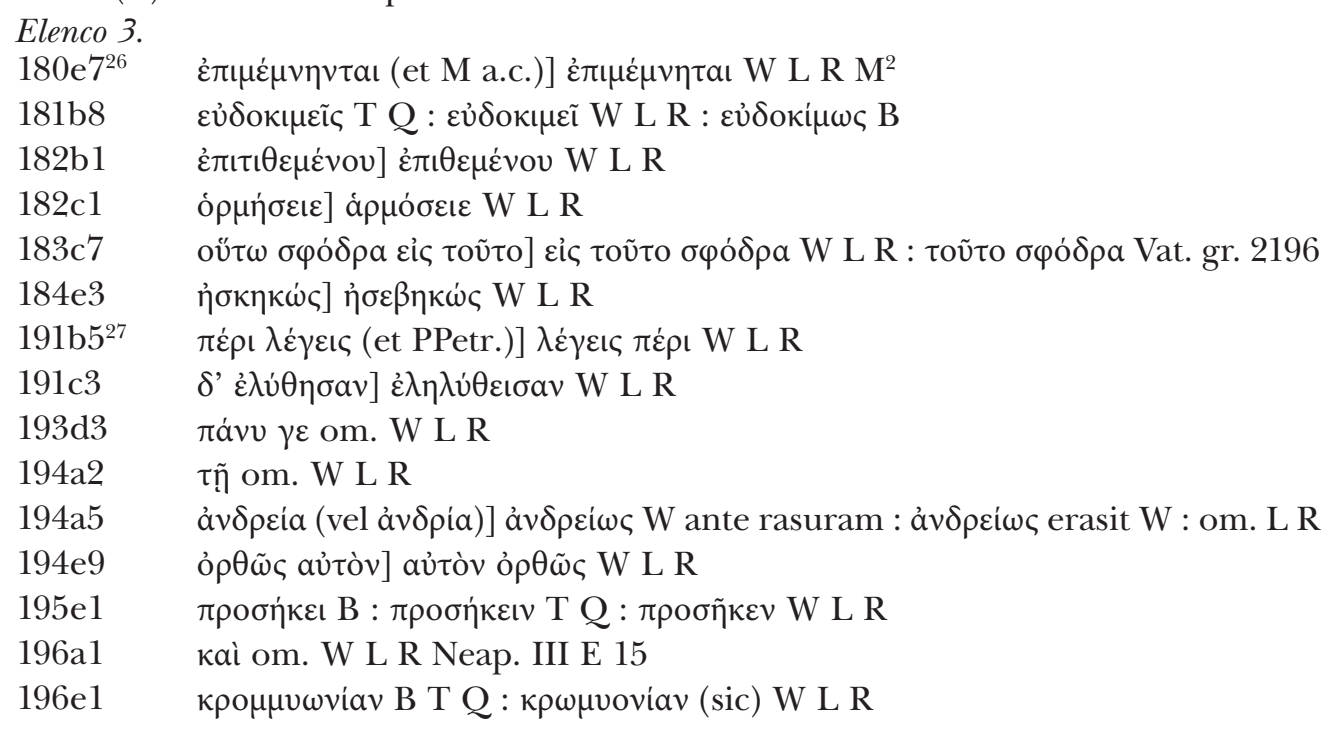

23 Si rinvia agli studi e alle trattazioni di Boter (1987); Brockmann (1992: spec. pp. 248-252). Diversa la posizione di Martinelli Tempesta (1997: pp. 213-278) che esclude, per il Liside, l'esistenza di una fonte comune a T e W; cfr. però le recensioni di Slings (1999: p. 490) e Vendruscolo (2000: pp. 118-120). Cfr. anche Slings (1998: p. 213). Nel Teage B, T e W deriverebbero indipendentemente l'uno dall'altro da un archetipo: cfr. Joyal (1998: pp. 40-45). Come detto sopra (p. 42), non si analizzano in questa sede i rapporti di Q con B, T e W nel Lachete; per il Carmide cfr. Murphy (1990: pp. 331-332, 335-338). Su P nel Simposio cfr. Brockmann (1992: pp. 153-155).

24 Murphy (1994: p. 4, n. 8).

25 Qualche errore si ritrova in apografi appartenenti agli altri rami. Altre lezioni peculiari di W e del suo ramo sono indicate in Carlini (1992: p. 25).

$26 \mathrm{M}=$ Cesena, Biblioteca Malatestiana, Plut. D. XXVIII 4. Con $\mathrm{M}^{2}$ ci si riferisce alla diorthosis condotta sul codice, sulla quale cfr. Martinelli Tempesta 1997: pp. 108, n. 339, pp. 109-111, cui si rinvia anche per ulteriori approfondimenti bibliografici. Per una descrizione del Malatestiano e soprattutto per la sua collocazione stemmatica nei quadri tradizionali di altri dialoghi, cfr. ad esempio per la Repubblica Boter 1989: pp. 27-28, 113-118, 120-123, 225-231; per il Simposio Brockmann 1992: pp. 18, 209-219; per Crizia e Timeo Jonkers 2017: pp. 46-47, 303-307, 341-344.

27 Le informazioni su PPetr. (= PPetrie II 50) e POxy 228 sono desunte da Carlini (1999b) e (1999a). 


\begin{tabular}{|c|c|}
\hline $198 \mathrm{e} 1$ & $\left.\dot{\eta} \gamma \varepsilon \omega \rho \gamma^{\prime} \alpha\right]$ $\gamma \varepsilon \omega \rho \gamma^{\prime} \mathbf{a}$ W L R \\
\hline $199 \mathrm{~d} 7$ & $\sigma \grave{u}] \sigma o t$ W L R \\
\hline $\mathrm{b} 4$ & $\dot{\varepsilon} \pi \alpha \nu \circ \rho \theta \dot{\omega} \sigma \varepsilon \sigma \theta \alpha \mathrm{l}] \dot{\varepsilon} \pi \alpha \nu o \rho \theta \dot{\omega} \sigma \alpha \sigma \theta \alpha \mathrm{l}$ W L R \\
\hline $0 \mathrm{~d} 1$ & 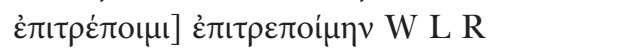 \\
\hline
\end{tabular}

\section{Lobcoviciano e il Vaticano gr. 1029}

Alla famiglia di W appartengono, pertanto, altri due manoscritti, il cosidetto Lobcoviciano (Nelahozeves, Roudnická Lobkowiczká knihovna, VI Fa 1; La. pp. 845-869) e il Vat. gr. 1029 (La. ff. 27v-38r). ${ }^{28}$

La datazione di L è stata oggetto di un vivo dibattito che ha coinvolto paleografi e filologi, a cominciare dalla proposta di L. Perria di attribuire L all' 'Anonimo k', retrodatandolo quindi alla fine del sec. XI (o al più tardi agli inizi del XII); il catalogo presenta l'indicazione «Saec. XII ex. (?)». ${ }^{29}$ L'opinione oggi prevalente, tuttavia, colloca il manoscritto nel sec. XIV: N. G. Wilson, ad esempio, richiamando la datazione al sec. XIV avanzata da L. A. Post, propone che si possa trattare di una scrittura 'di imitazione'. ${ }^{30}$ Sotto un profilo prettamente critico-testuale, L si rivela apografo di $\mathrm{W},{ }^{31}$ come già ipotizzato da Schanz nel 1877, mentre L. Perria lo riteneva copiato dallo stesso modello di W. ${ }^{32}$

28 Per una descrizione dei due codici cfr. ad esempio: per L Boter (1989: pp. 49-50); Jonkers (2017: pp. 71-72); per R Boter (1989: pp. 52-53); Jonkers (2017: p. 77). Per L si veda anche la scheda catalografica di Olivier \& Monégier du Sorbier (1983: pp. 97-103), non affidabile tuttavia per la datazione del codice (v. sotto). Sulla storia del codice cfr. anche Carlini (1992: pp. 22-24) e sulla sua segnatura cfr. di recente Vancamp (2010: p. 42). Per R non è disponibile un catalogo a stampa. Petrucci (2014) fornisce una dettagliata descrizione di questo codice, della sua storia e del suo ruolo all'interno della tradizione. Come dimostrato da G. Boter (1988: pp. 215-216), il Lobcoviciano può difficilmente essere identificato con il codice utilizzato da Janus Cornarius nelle sue Eclogae decem, dove egli afferma di essersi servito di un manoscritto appartenente alla famiglia Lobkowicz.

29 Perria (1985-1986: pp. 82-90); (1992), dove è contemplata anche la possibile collocazione all'inizio del sec. XII; Olivier \& Monégier du Sorbier (1983: p. 97). Cfr. anche per una sintesi delle varie proposte di datazione ad esempio Berti (1996: pp. 99-101); Murphy (2002: p. 155); Pérez Martín (2005: p. 125). Liidentificazione della mano di L proposta da L. Perria è stata accolta favorevolmente p.e. da Eleuteri (1986: p. 549; opinione riaffermata in Eleuteri 1993: pp. 455-456, 458); Prato (1993: p. 281).

30 Wilson (1985: p. 176): «one is strongly tempted to suggest that the scribe is producing a clever imitation of a middle Byzantine script for the benefit of an Italian Renaissance scholar. If this view is eventually proved to be correct, the case will not be unique; there is another well known example in MS Pal.gr. 186, a copy of Apollonius Rhodius which used to be assigned to the 11th century but can be confidently placed in the 15th»; Post (1934: p. 90). Cfr. Pérez Martín (2005: pp. 126-131). Sul Pal. gr. 186 cfr. Irigoin (1981). Esempi di manoscritti 'mimetici' in Prato (1979); sul fenomeno cfr. anche i contributi ricordati da Carlini (1992: pp. 31-33, n. 62), tra cui Prato (1981).

31 Cfr. ad esempio Carlini (1992: pp. 26 -32, che esamina anche il rapporto tra L e W3). Si rinvia a Jonkers (2017: pp. 71-72) per una rassegna bibliografica sul rapporto tra W e L in diversi dialoghi. Le considerazioni precedentemente espresse (cfr. Brockmann 1992: pp. 28, 238) sono state revocate da Brockmann, che ora ritiene che un «guter [...] Imitator» abbia vergato il Lobcoviciano (1998: p. 662, n. 27). Vancamp (1995: pp. 44-46; 1996a: p. 13; 1996b: p. 43) conferma che L deriva da W nell'Ippia Maggiore e nell'Ippia Minore, accogliendo tuttavia l'ipotesi dell'identità di mano di L e W. Agli atti del seminario sull'inquadramento di L all'interno della tradizione platonica, tenutosi al Dipartimento di Filologia classica dell'Università di Pisa il 9 e 10 marzo 1992, si rinvia per ulteriori approfondimenti sui piani filologico e paleografico: 
R è stato vergato da due mani: R1: ff. 1-352 della prima parte; R2: ff. 353-487 della prima parte e seconda parte (ff. 1-517). Il codice viene collocato alla fine del sec. XIII o agli inizi del XIV, datazione, quest'ultima, preferita anche da L. A. Post (1934: p. 78) e compatibile con la derivazione di $\mathrm{R}$ da L (v. sotto), che escluderebbe quindi altre proposte (secc. XI, XII). ${ }^{33}$

Tutti gli errori di W sono copiati da L (v. Elenco 3) che ne introduce anche altrii, ${ }^{34}$ presenti anche in $\mathrm{R}$, come i seguenti:

Elenco 4.

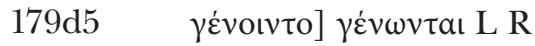

$179 \mathrm{e} 1 \quad$ kaì om. L R

181e2

182b6 غ̇ं

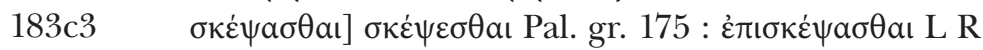

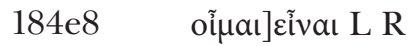

$193 \mathrm{e} 2 \quad \gamma \alpha \dot{\rho} \rho$ om. L R

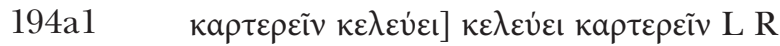

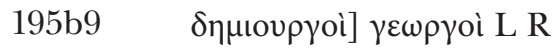

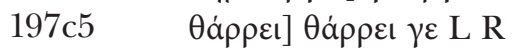

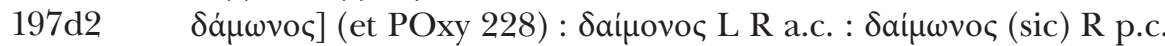

198b8 ià om. L R

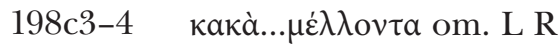

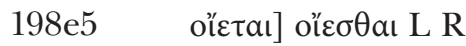

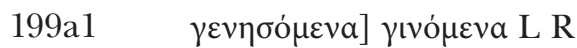

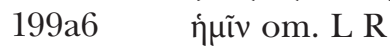

$199 \mathrm{e} 9 \quad \gamma \varepsilon$ om. L R

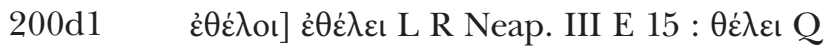

Una relazione fra i tre codici è quindi evidente, rimane da chiarire di che natura essa sia e se possa spiegarsi con l'ipotesi avanzata da M. Schanz secondo cui L sarebbe l'intermediario fra W e R, o con quella proposta da L. A. Post, che considerava R e L gemelli. ${ }^{35}$

Il Lobcoviciano (1992). Indizi materiali della dipendenza diretta di $\mathrm{L}$ da $\mathrm{W}$ (salto di righe e di folia, etc.) sono stati individuati p.e. da Jonkers (2017: p. 235); Berti (1992: pp. 39 sul pinax, 46-50 sui danni, come i margini tagliati, in W); Berti (1996: p. 52).

32 Schanz (1877: p. 62); Perria (1985-1986: p. 85). Elementi critico-testuali e paleografici a sostegno della diversità di mano dei due manoscritti e della dipendenza di L da W in Murphy (1992b). Le caratteristiche paleografiche di L sono descritte anche in Wilson (1994). Sull'hyphen nei codici cfr. Murphy (1995b).

33 Su R1 e R2 cfr. Peréz Martin (2005: p. 129), secondo cui R1 sarebbe «l'esperto ed abile calligrafo che imitò alla perfezione la scrittura di W due secoli dopo la sua copia», ossia il copista di L. Per alcune proposte di datazione cfr. Boter (1989: p. 52). Sulla derivazione, verosimilmente diretta, di R da L in diversi dialoghi si rinvia alle indicazioni bibliografiche in Jonkers (2017: p. 77).

34 Anche Murphy (1992b: p. 101 e n. 12) osserva questa situazione, senza tuttavia riportare gli errori. Per alcune lezioni che escludono la dipendenza di W3 da L cfr. Murphy (1992b: p. 101).

35 Schanz (1877: pp. 100-102); Post (1934: pp. 30-35). Schanz (1877: p. 61) ricordava tra gli esempi a sostegno della dipendenza di $\mathrm{R}$ da $\mathrm{W}$ anche un passo del Lachete: a $194 \mathrm{a} 5 \mathrm{R}$ omette ảv $\delta \rho \varepsilon \dot{\alpha}$, parola evanida in W. Berti (1992: p. 60) ha confermato l'omissione del termine anche in L. 
Le lezioni (inferiori) presenti in $\mathrm{L}$, ma non in $\mathrm{R}$, sono poche:

Elenco 5.

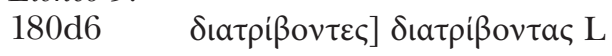

$198 \mathrm{c} 4$ бù $\delta \dot{\varepsilon}]$ oủ $\delta \dot{\varepsilon} \mathrm{L}$

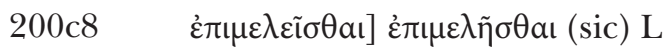

In questi casi $\mathrm{R}$ ripristina la lezione corretta non necessariamente perché la legga in W. Si potrebbe supporre un'attività di contaminazione con un altro codice (o più di uno), oppure, trattandosi di errori facilmente 'reversibili', di buone congetture. ${ }^{36}$

Analogamente il copista può aver facilmente congetturato le - poche - lezioni corrette condivise da $\mathrm{R}$ e da codici appartenenti ad altre famiglie:

Elenco 6.

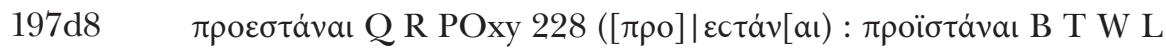

$199 \mathrm{~b} 10^{37} \pi \dot{\rho} \rho \mathrm{M}^{2} \mathrm{R}: \pi \varepsilon \rho \grave{\mathrm{B}} \mathrm{T}$ W L Q

Numerose sono le lezioni singolari di R. Tra di esse ricordo le più significative: ${ }^{38}$

\begin{tabular}{|c|c|}
\hline $179 \mathrm{e} 4$ & 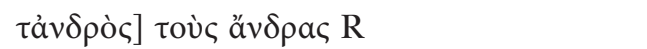 \\
\hline 180b1-2 & 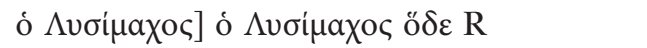 \\
\hline $180 \mathrm{~d} 3$ & $\sigma v v \delta ı \alpha \tau i \beta \varepsilon เ v] \delta ı \alpha \tau i \beta \varepsilon เ v \mathrm{R}$ \\
\hline $181 \mathrm{e} 1$ & $\omega \dot{\omega} \varphi \dot{\varepsilon} \lambda \iota \mu \nu . . . \varepsilon \dot{\varepsilon} \pi \dot{i} \sigma \alpha \sigma \theta \alpha \iota$ om. R \\
\hline $182 b 3$ & $\delta^{\prime}$ oủ $\delta \dot{\varepsilon}$ útò] $\delta^{\prime}$ útò $\mathrm{R}$ \\
\hline $183 c 6$ & $\dot{\varepsilon} \pi ı \tau \eta \delta \varepsilon v \sigma \alpha \dot{v} \nu \tau \omega \nu] \dot{\varepsilon} \pi ı \tau \eta \delta \varepsilon v \mu \alpha \dot{\tau} \omega \nu \mathrm{R}$ \\
\hline $183 \mathrm{~d} 2$ & $\dot{\varepsilon} \tau \dot{\varepsilon} \rho \omega \theta \mathrm{\iota}$ om. R \\
\hline 184b1 & 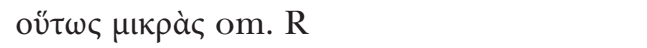 \\
\hline $184 d 1$ & $\beta o v \lambda \eta \dot{\eta}] \sigma v \mu \beta$ ov $\lambda \dot{\eta} \mathrm{R}$ \\
\hline $184 d 4$ & 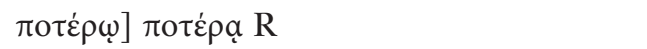 \\
\hline $185 \mathrm{a} 1$ & 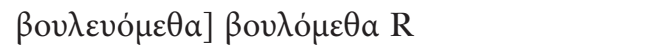 \\
\hline $186 \mathrm{~d} 6$ & $\sigma \varepsilon] \gamma \varepsilon \mathrm{R}$ \\
\hline $186 \mathrm{e} 4$ & $\delta \grave{~ o m . ~ R ~}$ \\
\hline $187 \mathrm{c} 8$ & kaì om. $\mathrm{R}$ \\
\hline $188 \mathrm{e} 5$ & $\left.\delta^{\prime} \dot{\varepsilon} \gamma \grave{\omega} \tau \tilde{\omega} \nu\right] \delta \grave{\varepsilon} \tau \tilde{\omega} \nu \mathrm{R}$ \\
\hline $189 \mathrm{~b} 7$ & $\mu \eta \delta \dot{\varepsilon} v]$ oủ $\delta \grave{\varepsilon} v \mathrm{R}$ \\
\hline $189 c 3$ & $\varepsilon \varepsilon^{\prime} \gamma \omega \varepsilon$ om. R \\
\hline $189 c 4-5$ & 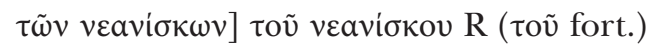 \\
\hline $189 c 8$ & àv om. R \\
\hline $189 \mathrm{e} 4$ & $\tau \omega] \tau] \mathrm{R}$ \\
\hline
\end{tabular}

36 Correzioni introdotte per congettura sono state individuate anche in Liside, Ippia Maggiore, Ippia Minore, Repubblica e Timeo: cfr. rispettivamente Martinelli Tempesta (1997: p. 139); Berti (1992: pp. 53-54); Vancamp (1996a: p. 44); Boter (1989: p. 166); Jonkers (2017: p. 236). Murphy (1990: p. 333) propone, per il Carmide, che gli interventi siano dovuti a congettura e contaminazione.

$37 \mathrm{Su} \mathrm{M} \mathrm{M}^{2}$ cfr. n. 26

38 A $196 \mathrm{~b} 8$ la coincidenza con Neap. è probabilmente poligenetica. 


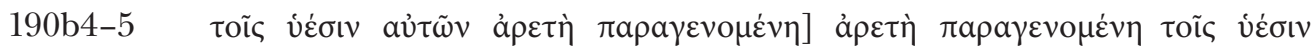

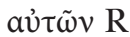

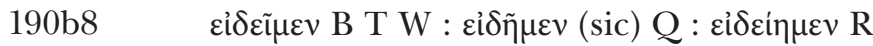

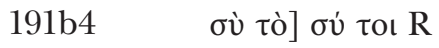

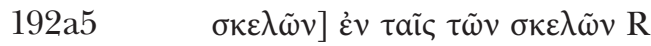

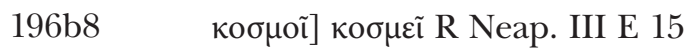

197c3 oľctal] हैotke R

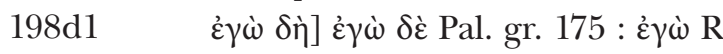

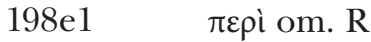

199e6 om. Ẽ̃vaı R

Si individuano dunque più le lezioni separative di $\mathrm{R}$ nei confronti di L che viceversa, il che esclude la derivazione di L da R. A ragione E. Berti (1992: pp. 51-52) ammoniva sulla necessità di prove 'materiali' per definire in modo univoco la relazione che intercorre tra i due codici. Nel Lachete non si riscontrano evidenze di questo tipo, individuate per altri dialoghi, ${ }^{39}$ tuttavia le lezioni presentate, in particolare il gran numero di congetture peculiari di R e il minor numero di errori, per lo più banali, di L, sembrano confermare la discendenza di $\mathrm{R}$ da $\mathrm{L} .{ }^{40}$

\section{Bibliografia}

de Andrés, G. (1965). Catálogo de los Códices Griegos de la Real Biblioteca de El Escorial, II: Códices 179-420. Madrid: Impr. Helénica.

Berti, E. (1992). Cinque manoscritti di Platone (Vind. W, Lobc., Vat. R, Laur. C.S. 54 e 78). In Lobcoviciano (pp. 37-74).

Berti, E. (1996). Ancora sul codice Lobcoviciano di Platone. In M. S. Funghi (Ed.), O $\Delta \mathrm{OI} \Delta \mathrm{IZH} \Sigma \mathrm{IO} \Sigma$. Le vie della ricerca. Studi in onore di Francesco Adorno (pp. 95-107). Firenze: Olschki.

Berti, E. (2001). Marsilio Ficino e il testo greco del Fedone di Platone. In J. Hamesse (Ed.), Les traducteurs au travail. Leurs manuscrits et leurs méthodes. Actes du Colloque international organisé par le "Ettore Majorana Centre for Scientific Culture" (Erica, 30 september - 6 october 1999) (pp. 349-425). Turnhout: Brepols.

Boter, G. (1987). The Vindobonensis W of Plato. Codices manuscripti, 13(4), 144-155.

Boter, G. (1988). The Codex Hassensteinianus of Plato. Revue d'Histoire des Textes, 18, 215-218.

Boter, G. (1989). The Textual Tradition of Plato's Republic. Leiden - New York - København - Köln: Brill.

Brockmann, C. (1992). Die handschriftliche Überlieferung von Platons Symposion. Wiesbaden: Reichert. Brockmann, C. (Rev.). (1998). E. A. Duke, W. F. Hicken, W. S. M. Nicoll, D. B. Robinson, \& J. C.

39 Filebo: Berti (1992: p. 52); Ippia Maggiore: Vancamp (1995: p. 47) (il caso dei ff. 371v-372r è anticipato in Berti 1992: pp. 52-53); Ippia Minore: Vancamp (1996a: pp. 43-44, spec. n. 41); Timeo: Jonkers (2017: p. 236).

40 La medesima situazione in altri dialoghi: rassegna in Jonkers (2017: p. 77) e, per l'analisi nella tradizione del Timeo, Jonkers (2017: pp. 236-237). 
G. Strachan, Platonis Opera (tom. 1; 1995). Gnomon, 70(8), 657-665.

Brumbaugh, R. S. (1990). Plato Manuscripts: Toward a Completed Inventory. Manuscripta, 34, 114-121.

Brumbaugh, R. S., \& Wells, R. (1968). The Plato Manuscripts: A New Index. New Haven - London: Yale University Press.

Burnet, J. (Ed.) (1909²). Platonis Opera. Tomus III tetralogias V-VII continens. Oxonii: Clarendon Press. Carlini, A. (Ed.) (1964). Platone. Alcibiade, Alcibiade secondo, Ipparco, Rivali. Torino: Boringhieri.

Carlini, A. (1972). Studi sulla tradizione antica e medievale del Fedone. Roma: Edizioni dell'Ateneo.

Carlini, A. (1992). Le vicende storico-tradizionali del Vind. W e i suoi rapporti con il Lobcoviciano e il Ven. Gr. Z 185. In Lobcoviciano (pp. 11-35).

Carlini, A. (1999a). POxy 228. In F. Adorno et al. (Eds.), Corpus dei papiri filosofici greci e latini (CPF), I: Autori Noti, Vol. 1, 3: Nicolaus Damascenus - Platonis Fragmenta (pp. 113-118). Firenze: Olschki.

Carlini, A. (1999b). PPetrie II 50. In F. Adorno et al. (Eds.), Corpus dei papiri filosofici greci e latini (CPF), I: Autori Noti, Vol. 1, 3: Nicolaus Damascenus - Platonis Fragmenta (pp. 100-113). Firenze: Olschki.

Dodds, E. R. (Ed.) (1959). Plato: Gorgias. A Revised Text with Introduction and Commentary. Oxford: Clarendon Press.

Duke, E. A. (1991). The place of Parisinus graecus 1813 in the tradition of the Phaedo. Revue d'Histoire des Textes, 21, 243-256.

Duke, E. A., Hicken, W. F., Nicoll, W. S. M., Robinson, D. B., \& Strachan, J. C. G. (Eds.). (1995). Platonis Opera. Tomus I tetralogias I-II continens. Oxonii: Clarendon Press.

Eleuteri, P. (Rev.). (1986). J.-M. Olivier, \& M-A. Monégier du Sorbier, Catalogue des manuscrits grecs de Tchécoslovaquie (1983). Gnomon, 58(6), 548-549.

Eleuteri, P. (Rev.). (1993). F. Adorno, Studi su codici e papiri filosofici. Platone, Aristotele, Ierocle (1992). Rivista di Filologia e di Istruzione Classica, 121(4), 455-459.

Hunger, H. (1957). Katalog der griechischen Handschriften der Österreichischen Nationalbibliothek. Supplementum Graecum. Wien: Notring der wissenschaftlichen Verbände Österreichs.

Hunger, H., \& Hannick, C. (1994). Katalog der griechischen Handschriften der Österreichischen Nationalbibliothek, 4: Supplementum Graecum. Wien: Hollinek.

Irigoin, J. (1981). Une écriture d'imitation: le Palatinus Vaticanus graecus 186. Illinois Classical Studies, 6(2), 416-430.

Jonkers, G. (2017). The Textual Tradition of Plato's Timaeus and Critias. Leiden - Boston: Brill.

Joyal, M. (1998). The textual tradition of [Plato], Theages. Revue d'Histoire des Textes, 28, 1-54.

Král, J. (1892). Über den Platocodex der Wiener Hofbibliothek suppl. phil. gr. 7. Wiener Studien, 14, 161-208.

Lobcoviciano (1992). Il Lobcoviciano di Platone sotto analisi paleografica e filologica. In Studi su codici e papiri filosofici. Platone, Aristotele, Ierocle (pp. 7-143). Firenze: Olschki.

Maass, E. (1884). Observationes palaeographicae. In Mélanges Graux. Recueil des travaux d'érudition classique dédié a la mémoire de Charles Graux (pp. 749-776). Paris: Ernest Thorin.

Marg, W. (Ed.) (1972). Timaeus Locrus. De natura mundi et animae. Leiden: Brill.

Manfrin, F. (2017). Studi sulla tradizione manoscritta dell' Eutifrone di Platone: la terza famiglia. Revue d'Histoire des Textes, 12, 1-34.

Martinelli Tempesta, S. (1997). La tradizione testuale del Liside di Platone. Firenze: La Nuova Italia. 
Mazal, O. (1981). Byzanz und das Abendland. Katalog einer Ausstellung der Handschriften- und Inkunabelsammlung der Österreichischen Nationalbibliothek. Wien: Österreichische Nationalbibliothek.

Menchelli, M. (1991). Il Vaticano Palatino gr. 173 (P) di Platone e il Parigino gr. 1665 di Diodoro. Bollettino dei Classici, III. s., 12, 93-117.

Menchelli, M. (2007). L’Anonimo Г del Laur. Plut. 85.6 (Flor) e il Vind. Suppl. gr. 39 (F). Appunti sul "gruppo w" della tradizione manoscritta di Platone e su una "riscoperta" di età paleologa. Medioevo greco, 7, 159-182.

Mioni, E. (1976). Bessarione scriba e alcuni suoi collaboratori. In Miscellanea marciana di studi bessarionei (a coronamento del V Centenario della donazione nicena) (pp. 263-318). Padova: Antenore.

Moreschini, C. (1965). Studi sulla tradizione manoscritta del Parmenide e del Fedro di Platone. Annali della Scuola Normale Superiore di Pisa. Lettere, Storia e Filosofia, II s., 34(1-2), 169-185.

Murphy, D. J. (1990). The Manuscripts of Plato's Charmides. Mnemosyne, IV s., 43(3-4), 316-340.

Murphy, D. J. (1992a). The Independence of Parisinus Graecus 1813 in Plato's Phaedrus, Hipparchus and Alcibiades II. Mnemosyne, IV. s., 45(3), 312-332.

Murphy, D. J. (1992b). The Plato Manuscripts W and Lobcovicianus. Greek, Roman, and Byzantine Studies, 33(1), 99-104.

Murphy, D. J. (1994). Parisinus gr. 1813 and Its Apographa in Plato's Laches. Mnemosyne, IV. s. 47(1), $1-11$.

Murphy, D. J. (1995a). Contribution to the History of Some Manuscripts of Plato. Rivista di Filologia e di Istruzione Classica, 123, 155-168.

Murphy, D. J. (1995b). Hyphens in Greek Manuscripts. Greek, Roman, and Byzantine Studies, 36(3), 293-314.

Murphy, D. J. (2002). The Basis of the Text of Plato's Charmides. Mnemosyne, 55(2), 131-158.

Murphy, D. J., \& Nicoll, W. S. M. (1993). Parisinus Graecus 1813 in Plato's Cratylus. Mnemosyne, IV. s., 46(4), 458-472.

Nicoll, W. S. M. (1966). Some Manuscripts of Plato's Apologia Socratis. The Classical Quarterly, n.s., 16(1), 70-77.

Olivier, J.-M., \& Monégier du Sorbier, M.-A. (1983). Catalogue des manuscrits grecs de Tchécoslovaquie. Paris: Centre National de la Recherche Scientifique.

Pérez Martín, I. (2005). Estetica e ideologia nei manoscritti bizantini di Platone. Rivista di Studi Bizantini e Neoellenici, n.s., 42, 113-135.

Perria, L. (1983-1984). Il codice W di Platone e il Vat. gr. 407. Rivista di Studi Bizantini e Neoellenici, n.s., 20-21, 93-101.

Perria, L. (1985-1986). Note paleografiche, II: Altre testimonianze sul copista di W. Rivista di Studi Bizantini e Neoellenici, n.s., 22-23, 82-90.

Perria, L. (1990). Nuovi frammenti del copista del codice W di Platone nel Vat. gr. 2646. Bollettino della Badia Greca di Grottaferrata, n.s., 44, 127-138.

Perria, L. (1992). A proposito del codice L di Platone. Problemi di datazione e di attribuzione. In Lobcoviciano (pp. 103-136).

Petrucci, F. M. (2014). Il Vat. gr. 1029 di Platone: struttura codicologica e dinamiche di allestimento. Segno e Testo, 12, 333-364.

Pinakes. Textes et manuscrits grecs, banca dati dell'Institut de Recherche et d'Histoire des Textes (CNRS, Paris) [online; https://pinakes.irht.cnrs.fr/; acessed 28.09.2021]. 
Pontani, F. (1997). Per la tradizione antica del Lachete di Platone: PPetrie II, 50 e POxy 228. Studi Classici e Orientali, 45, 99 -126.

Post, L. A. (1934). The Vatican Plato and Its Relations. Middletown (Connecticut): American Philological Association.

Prato, G. (1979). Scritture librarie arcaizzanti della prima età dei Paleologi e i loro modelli. Scrittura e Civiltà, 3, 151-193.

Prato, G. (1981). La produzione libraria in area greco-orientale nel periodo del regno latino di Costantinopoli (1204-1261). Scrittura e Civiltà, 5, 105-147.

Prato, G. (1993). Due postille paleografico-codicologiche. In F. Berger, C. Brockmann, G. De Gregorio, M. I. Ghisu, S. Kotzabassi, \& B. Noack (Eds.), Symbolae Berolinenses für Dieter Harlfinger (pp. 279-291). Amsterdam: Hakkert.

Schanz, M. (1877). Über den Platocodex der Markusbibliothek in Venedig Append. Class. 4 Nr. 1. Leipzig: Bernhard Tauchnitz.

Slings, S. R. (Rev.). (1998). B. Vancamp, Platon. Hippias maior - Hippias minor (1996). Mnemosyne, IV. s., 51(5), 611-616

Slings, S. R. (Rev.). (1999). S. Martinelli Tempesta, La tradizione testuale del Liside di Platone (1997). Mnemosyne, IV. s., 52(4), 489-492.

Stevenson, H. (1885). Codices manuscripti Palatini Graeci Bibliothecae Vaticanae. Romae: ex Typographeo Vaticano.

Vancamp, B. (1995). La tradition manuscrite de l'Hippias majeur de Platon. Revue d'Histoire des Textes, 25, 1-60.

Vancamp, B. (Ed.) (1996a). Platon. Hippias maior - Hippias minor. Stuttgart: Franz Steiner.

Vancamp, B. (1996b). La tradition manuscrite de l'Hippias mineur de Platon. Revue Belge de Philologie et d'Histoire, 74(1), 27-55.

Vancamp, B. (2010). Untersuchungen zur handschriftlichen Überlieferung von Platons Menon. Stuttgart: Franz Steiner.

Vendruscolo, F. (2000). Storia del testo di Platone: A proposito di uno studio recente (Martinelli Tempesta, 1997, Rev.). Rivista di Filologia e di Istruzione Classica, 128(1), 110-121.

Wilson, N. G. (1962). A list of Plato manuscripts. Scriptorium, 16, 386-395.

Wilson, N. G. (1985). Codices Bohemiae Graeci (Olivier \& Monégier du Sorbier, 1983, Rev.). The Classical Review, n.s., 35(1), 175-176.

Wilson, N. G. (1994). The Prague Manuscript of Plato. Studi Classici e Orientali, 44, 23-32. 
Maria Vittoria Curtolo, M. A. / mariavittoria.curtolo@phd.unipi.it

Dipartimento di Filologia, Letteratura e Linguistica

Università di Pisa

Palazzo Matteucci - Piazza Evangelista Torricelli 2, 56126 Pisa, Italia

Centre for the Study of Manuscript Cultures

Universität Hamburg

Warburgstraße 26, 20354 Hamburg, Deutschland 
